\title{
An Evolutionary Perspective on Population Growth
}

\author{
ROBERT KELLY
}

Doctor of Medicine

Amsthospitalet i Vordingborg

Vordingborg, Denmark

\begin{abstract}
This article is a preliminary attempt to evaluate the effect that evolution has on fertility. First, the conditions necessary for an evolutionary effect are discussed, the most important condition being the existence of fertility-enhancing traits (not necessarily genetic) which can be passed from parent to child. Next, two mathematical models are discussed which give insight into the relation between evolution and fertility. The models yield a crude approximation relating the correlation $(r)$ between number of siblings and number of children born to women in a given population to a subsequent evolution-related rise in fertility in the same population over one generation. The approximation is evaluated using the value of $r$ as determined from a 1981 study sample of Swedish-born women. Finally, the possibilities of long-term fertility predictions and control of population growth are discussed.
\end{abstract} Sweden

Keywords: evolution, population growth, fertility, traits, sibship, siblings, children, correlation,

\section{Introduction}

When Thomas Malthus stated his principle of population at the turn of the nineteenth century, the weight of evidence from contemporary population trends supported his theory. Since then we have learned in the twentieth century that socioeconomic factors involved in a "fertility transition" can lead to even lower than replacement level fertility in spite of good health, low mortality, and an abundant food supply. Numerous countries have yet to experience the fertility transition and a question for the twenty-first century is whether those countries will soon undergo the transition or instead remain stubbornly resistant, causing problems with overpopulation. Even developed nations may experience problems with too low or too high fertility in the next century.

In order to prepare ourselves for the challenges of the next century it is of value to learn about the factors governing population growth. With increased understanding we may be able to predict and perhaps to influence population growth. Recent demographic studies which deal with this subject try to identify relationships between various societal parameters (such as extent of urbanization, education, availability of birth control, etc.) and observed population growth. The results of similar studies from around the world are then compared and generalizations drawn which hopefully can be used 
to predict and understand trends in new situations. An important factor yet to be considered in such studies is the effect of evolution, the subject of this paper.

Every new generation represents the children of people who succeed in reproducing themselves. Those having large families are overrepresented in the next generation while those abstaining from having children are not represented at all. In a perfectly homogeneous population, this difference with regard to offspring has no evolutionary effect. However, if individuals in a population vary with respect to certain traits which affect their tendency to have children (children who survive and are capable of reproducing once they reach maturity) in an additive fashion and these traits can to some extent be passed on to their children, then those traits which decrease the number of offspring will decrease while those which increase the number of offspring will increase in prevalence in the next generation. These traits can be genetic or they can fall into the category commonly referred to by demographers as culture (attitudes, religions, belief systems, traditions, etc.).

Thus, if there are differences among individuals concerning their inherent tendency to have children (a reasonable assumption considering the wide range of physical and mental characteristics found among people in any given society in the world) and this tendency is due to inheritable traits (as discussed above), then even if all other nonevolutionary societal conditions governing fertility are held constant, we can expect an increase in fertility, thanks to evolution. Under varying conditions this evolutionary effect can also play a role, so naturally it is of interest to devise methods for verifying its existence and for measuring its size.

\section{Purpose}

The purpose of this study is to:

1. develop simple but realistic mathematical models to gain insight into evolutionary effects.

2. based on the models derive equations and approximations useful in gauging the size of the evolutionary effect, should one exist.

3. approximate the short-term effect of evolution in Sweden based on current data.

4. speculate concerning long-term evolutionary effects.

\section{Materials and methods}

Data were extracted from a larger study performed by Statistics Sweden in 1981 (Lyberg 1984). The sample for the study consisted of 5,000 randomly selected women born in Sweden 1936-1960 and living in Sweden in February, 1981. The dropout from the sample was 13 percent overall, with a slightly higher dropout for women in the upper age bracket $40-44$ (18 percent), particularly if they had no children ( 25 percent). The data were collected by a team of professional interviewers who visited the women at their homes.

Only data from women in the 39-44 age bracket are used for the purpose of this study. The number of women in this subsample is 659 . Information about the number of siblings is taken from question 20 and information about the number of children from question 63. Question 20 includes both the interviewee and any deceased siblings, but unfortunately it is not possible to determine to what extent half siblings are included here. Question 63 includes deceased children.

In addition to the 18 percent dropout for our age group, we can expect that be- 
tween 2 and 3 percent have dropped out due to premature death (Statistics Sweden 1982, 108). And, we can expect that less than 1 percent of the women in our study give birth to additional children between 1981 and the present time (Statistics Sweden 1990, 47).

\section{Results}

Figures 1 and 2 and Table 1 show data for sibship size and number of children for the individuals in our study sample. Mean values for sibship size and number of children are 3.61 and 2.08 , respectively. The corresponding standard deviations are 2.31 and 1.18 (evaluated using $\mathrm{N}-1=658$ ). The reason for the much larger mean and standard deviation for sibship size compared to family size is that sibship size is weighted toward larger values than is family size. For example, if two families have 2 and 6 children respectively, then the average sibship size for the children is 5 while the average family size for the parents is 4 .

Figure 1. Frequency distribution for number of children born to women in our study.

\section{Number of Children}

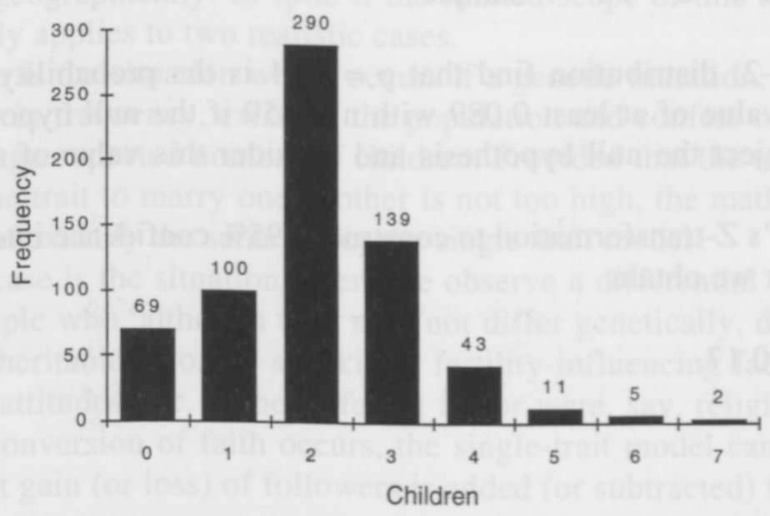

Fig u re 2. Frequency distribution for sibship size (number of siblings +1 ) of women in our study.

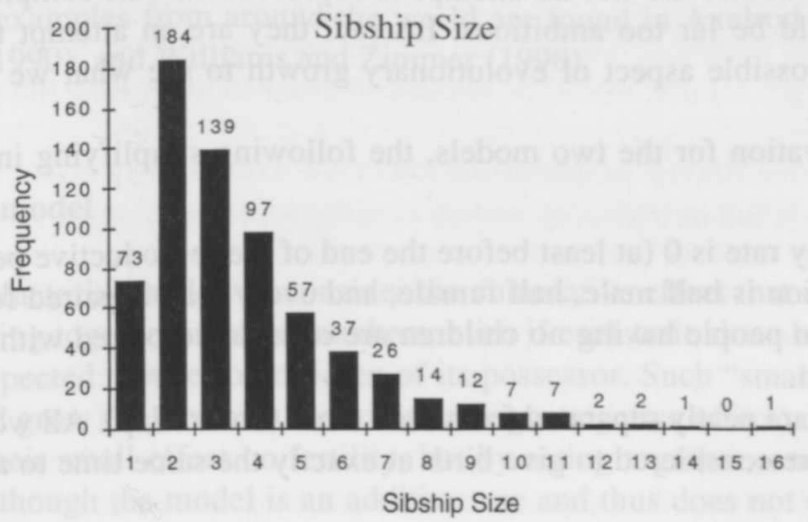


T a b le 1. Mean family size and frequency counts for each sibship size.

$\begin{array}{ccc}\text { Sibship size } & \text { Frequency } & \text { Mean family size } \\ 1 & 73 & 1.9589 \\ 2 & 184 & 1.9565 \\ 3 & 139 & 2.1871 \\ 4 & 97 & 2.0206 \\ 5 & 57 & 2.1579 \\ 6 & 37 & 2.4865 \\ 7 & 26 & 1.6538 \\ 8 & 14 & 2.0714 \\ 9 & 12 & 2.3333 \\ 10 & 7 & 2.2857 \\ 11 & 7 & 2.1429 \\ 12 & 2 & 1.5000 \\ 13 & 2 & 2.5000 \\ 14 & 1 & 4.0000 \\ 15 & 0 & 0.0000 \\ 16 & 1 & 7.0000\end{array}$

The calculated value of $r_{\text {sample }}$, the correlation between sibship size and family size for our sample, is 0.089 . We test the null hypothesis $(r=0$ for the population against $r>0$ ) with

$$
\mathrm{T}=\mathrm{r}_{\text {sample }} \sqrt{\mathrm{n}-2} / \sqrt{1-\mathrm{r}^{2} \text { sample }}
$$

and using a $t(n-2)$ distribution find that $\mathrm{p}=0.01$ is the probability that we would obtain an $r_{\text {sample }}$ value of at least 0.089 with $n=659$ if the null hypothesis were true. Therefore, we reject the null hypothesis and consider this value of $r_{\text {sample }}$ statistically significant.

Using Fisher's Z-transformation to construct a 95\% confidence interval for the population value of $r$ we obtain

$$
0.01<\mathrm{r}<0.17
$$

\section{Discussion}

In order to gain insight into what effects evolution can have, we consider two models, a single-trait model and a multifactorial model (detailed derivations available from author). These models are not an attempt to represent the full complexity of human life - that would be far too ambitious. Instead, they are an attempt to simplify and focus on one possible aspect of evolutionary growth to see what we can learn from the experiment.

In the derivation for the two models, the following simplifying implicit assumptions are made:

1. The mortality rate is 0 (at least before the end of the reproductive period).

2. The population is half male, half female, and every man is paired for life with one woman; even people having no children are considered paired with one another in some fashion.

3. Generations are neatly separated from each other (no overlap). All women in a given generation are considered to give birth at exactly the same time to all of their children. 
4. The population is large enough that actual parameters measured from the population differ insignificantly from the expected values calculated from the models.

Further, it is assumed that factors which influence fertility can be divided into inherent inheritable (including nongenetic) factors which can vary from individual to individual and environmental factors which include a certain element of randomness so that two people with exactly the same inherent characteristics in the same society will not necessarily have the same number of children.

The reason for assuming a zero mortality rate is that in developed nations it is fertility rather than mortality which limits population growth. The subject of this study is fertility, so there is no reason to complicate the picture by introducing mortality. The reason for assuming a constant generation length for all individuals is that the distribution of mother's age at childbearing only affects the time scale under which a population waxes or wanes, not the question of whether or not the population will increase, decrease, or stabilize.

\section{Single-trait model}

The single-trait model is derived with the idea that all people can be divided into two groups: those possessing a certain trait and those not, with marriage only occurring between members of the same group. The groups may coexist in the same place or be separated geographically. In spite if the limited scope of this model's premise, the model actually applies to two realistic cases.

The first case is the situation which occurs if a genetic mutation, dominant in expression and low in frequency, exists in the population and confers on its possessor a higher than average expected number of children. Provided that the tendency for people possessing the trait to marry one another is not too high, the mathematics for this situation are paradoxically the same as for the single-trait model.

The second case is the situation where we observe a differential fertility between two types of people who, although they may not differ genetically, do differ with respect to some inheritable (broadly speaking), fertility-influencing factor such as religion, traditions, attitudes, etc. If the differing factor were, say, religion and a certain amount of interconversion of faith occurs, the single-trait model can still apply provided that the net gain (or loss) of followers is added (or subtracted) from the fertility for each religion.

When this second case applies we observe an evolution of attitudes, ideas, or traditions rather than the classical evolution of genes. The idea that two such groups of people (having different fertilities) can coexist even in the same city is not so farfetched. Some examples from around the world are found in Axelrod (1990), Finnäs (1991), Jones (1990), and Williams and Zimmer (1990).

\section{Multifactorial model}

In the multifactorial model we consider the cumulative effect that numerous traits can have by acting together in large numbers, even if each trait alone has only a small effect on the expected number of children of its possessor. Such "small" traits by acting alone would grow (or diminish for traits having a negative effect) only very slowly because of their small effect on fertility, but by acting together can have a significant impact. Although the model is an additive one and thus does not directly imitate 
genetic transmission of traits, it still applies for both dominant traits in low prevalence and recessive traits in high prevalence.

\section{Short-term view}

One of the fruits of the models is that both show an approximately linear relationship between the value of $r$ and the evolution-related increase in fertility $(\Delta f)$ that we would expect over one generation. The single-trait model yields the approximation

$$
\Delta \mathrm{f} \approx \mathrm{k}_{\mathrm{s}} \mathrm{r}=1.3 \mathrm{r}
$$

where $\mathrm{k}_{\mathrm{s}}=2.31 * 1.18 / 2.08$, which is the product of the standard deviations for sibship size and number of children divided by the mean number of children from our sample. The multifactorial model yields a wide range of values for Df depending upon what assumptions are made concerning a number of parameters. One such parameter is the degree of matching of partners according to similar traits. The multifactorial model yields values for $\Delta \mathrm{f}$ which are close to or slightly higher than the values predicted by the single-trait model.

It would be nice if we could obtain an estimate of the effect of evolution in Sweden either via the multifactorial model or by simply evaluating (1) using $\mathrm{r}_{\text {sample }}$ in place of $\mathrm{r}$. However, there remain issues which must be clarified before such a quantification can take place.

First, it is not clear to what extent the models apply to the population of Sweden. They both assume the existence of inheritable fertility-enhancing traits which act consistently across generations, an assumption whose veracity remains to be demonstrated. Also not taken into account is how other fertility-influencing factors may modulate the evolutionary effect or the relationship between $r$ and an evolution-related rise in fertility.

Second, evolution is not the only factor which could cause a positive $\mathrm{r}$ value. Hypothetical models can even be constructed where the population is stable generation after generation in spite of a constant positive $r$ value (For example, consider a society where half of couples have three children, half have one child, and every person from a one-child family subsequently marries a person from a three-child family and has exactly one child). A more plausible reason for the positive value of $r_{\text {sample }}$ would be that the parents of some of the women in our study migrated to Sweden from countries which have much higher fertility than Sweden does. If it takes a few generations for the factors causing low fertility among Swedish women to take effect, then we would observe a positive correlation even in the absence of any long-term evolutionary effect.

Third, our $95 \%$ confidence interval for the value of $\mathrm{r}$ is too broad to be of practical value. A larger sample would be needed in order to determine the value of $\mathrm{r}$ more accurately.

Thus, a serious attempt at quantification of the evolutionary effect would at a minimum require a larger sample size and an investigation into factors which could influence $r_{\text {sample }}$. Such work is beyond the scope of this article. Other methods can also be developed to estimate the effect of evolution on fertility, and a challenge for future research is to find the most efficient and practical methods.

If we suppose for a moment that evolution is the only significant factor affecting the value of $r_{\text {sample }}$, it would be interesting to see what the single-trait model predicts for the effect of evolution over one generation (which for Sweden is somewhere between 25 and 30 years). Since our data were taken in 1981, we make projections for 
the year 2010. Beginning with our $95 \%$ confidence interval for $\mathrm{r}$ we apply (1) with an assumed error margin of $1 /(1 \pm 0.3)$ (error analysis not shown). On that basis the increase in fertility due to evolution (Df) is calculated to be between 0.01 and 0.31 by the year 2010 .

\section{Long-term view}

Studies which attempt long-term projections (Bourgeois-Pichat 1981; Frejka 1981; Statistics Sweden 1983, 1989; UNS 1982), share a common idea - that fertility in all regions of the world will level off near the replacement level by the latter half of the next century - but why this should be so is not clear. The reasons given are:

1. Any populations with non-trivial difference from replacement fertility would in the long run either disappear or continuously increase.

2. Looking at current trends in population growth we notice that fertility tends toward the replacement level.

The first argument is actually a quote from Frejka (1981). Another author (UNS 1982) expresses this idea with the words, “... because any other level of fertility, if maintained indefinitely, would eventually cause a population to expand to an improbable size or to decline to the point of disappearing." How this argument applies is not clear. If a country's fertility is high above the replacement level, what force is it that will cause the country's fertility to decline to the replacement level before population exceeds what the land can support? Apart from a fortuitous fertility transition or prudent government action there doesn't seem to be any guarantee against the individuals of a country collectively driving themselves to overpopulation ruin. In any case, the first argument does not explain why the fertility for a sparsely populated country like Sweden could not suddenly begin to increase within a few decades.

In answer to the second argument, let us ask: How much faith can we place in trends? Would a demographer at the beginning of the last century have been able to predict the fertilities of various countries in this century based on trend analysis? If the answer is no, then perhaps we can profit by an examination of underlying forces which could steer future developments in a direction opposite to current trends.

To get a feeling for what sort of long-term effects evolution can have let's imagine that there exists a pro-natalist group of people living within Sweden whose members only intermarry within the group. Let's further suppose that this group makes up one percent of the Swedish population and that the average family size for members of the group is 5.75 generation after generation, while that for the remaining 99 percent of the population is 1.75 . Under these circumstances, the single-trait model applies and the fertility from generation to generation can be calculated exactly. The results of this exercise are shown in Table 2.

T a b l e 2. An example of the evolutionary effect according to the single-trait model.

$\begin{array}{lcc}\text { Generation } & \text { Percentage with trait } & \text { Mean family size } \\ 0 & 1 & 1.80 \\ 1 & 3 & 1.88 \\ 2 & 10 & 2.14 \\ 3 & 26 & 2.81 \\ 4 & 54 & 3.91 \\ 5 & 79 & 4.93\end{array}$


This example is not as contrived as it might seem. In fact, it represents the situation in the municipality of Larsmo on the West Coast of Finland (Finnäs 1991). About 40 percent of Larsmo's 3,500 inhabitants are Laestadians, people who belong to the Lutheran Church and adhere to the revivalist movement Laestadianism, which is opposed to contraception. In spite of living in a modern Western society, the average family size among Laestadians in Larsmo has been approximately 6 (ranging from 5.47 to 6.75 ) for the last 25 years while it has been approximately 2 (ranging from 1.45 to 2.29) for Non-Laestadians living in the same area.

An examination of Table 2 shows that fertility increases almost imperceptibly during the first two generations and thereafter accelerates dramatically for generations 3,4 , and 5. This shows that although the short-term effects of evolution may be insignificant, a break-point may be reached where the evolutionary effect becomes great. Incidentally, the change in mean family size from generation 0 to 1 in this example is 0.08 which is close to the value of 0.12 obtained by using $r_{\text {sample }}$ in (1).

A natural question which arises is: If evolution is constantly acting to drive fertility upward, then how do we explain the observed declines in fertility for developed countries during the last hundred years or so? The answer is simple: Fertility went down for the reasons normally cited by demographers. Changes in society, predominantly socioeconomic, have made people less inclined to have children.

We could speculate that hundreds of years ago fertility was fine-tuned to a value which insured the long-term survival and maximum growth of the society. Too high a fertility was dangerous because of the difficulty in supporting such a rapid expansion and too low a fertility ran the risk of being left behind by more rapidly growing groups of people. In modern times conditions have changed suddenly so that evolution has not had time to once again bring fertility up to levels appropriate for maximum growth through the process of natural selection. Barring any new, fertility-suppressing changes in society, fertility may well begin to climb again thanks to evolution.

The question of whether or not evolution affects fertility is a subject for further study. Evolution is at work if and only if three conditions hold true:

1. There is variation among individuals within the society with respect to how many children they have and this variation depends to some extent on inherent traits of the parents.

2. The inherent traits are transmitted to some extent from parent to child.

3. Traits which tend to increase fertility do so consistently across generations.

Studies can be constructed to test these conditions for selected traits. These conditions can even be used as a basis for government programs to control population growth, should evolution prove to be an important factor influencing fertility.

\section{Conclusion}

Industrialized nations during the twentieth century have witnessed a decline in fertility attributable to certain concurrent socioeconomic changes. In view of current fertility trends and barring any dramatic socioeconomic upheavals, one might expect low fertility to continue for industrialized nations throughout the twenty-first century. However, even in such nations fertility may surprisingly rise to high levels during the next century because of the influence of evolution. For the same reason, countries awaiting the fertility transition may sluggishly remain at high fertility levels.

In this study two models are developed which shed light on the relationship between evolution and fertility and an attempt is made to estimate the impact which evolution will have on fertility over one generation for the country Sweden. The work here 
only scratches the surface of a mountain of possibilities for future research. Some important questions remain unanswered: Was the statistically significant value of $r_{\text {sample }}$ due to evolution or are other factors involved? Can we identify examples of the fertility-enhancing traits presupposed in the models? How do the approximations hold up when other fertility-influencing factors are changing rapidly? Can efficient methods for measuring the evolutionary effect be developed?

Predicting population growth is a bit like predicting the weather. So many factors act together in such a complicated fashion that only short-term predictions can be made with any credibility whatsoever. However, just as knowledge of the seasons and the earth's orbit around the sun can help to predict the weather, the study of evolution holds promise for helping with long-term projections. Evolution deserves a place in future demographic research.

\section{References}

Axelrod, Paul. 1990. Cultural and historical factors in the population decline of the Parsis of India. Population Studies 44(3):401-19.

Bourgeois-Pichat, Jean. 1981. Recent demographic change in Western Europe: an assessment. Population and Development Review 7(1):19-42.

Finnäs, Fjalar. 1991. Fertility in Larsmo: the effect of Laestadianism. Population Studies 45(2):339351.

Frejka, Tomas. 1981. Long-term prospects for world population growth. Population and Development Review 7(3):489-511.

Jones, Gavin W. 1990. Fertility transitions among Malay populations of Southeast Asia: puzzles of interpretation. Population and Development Review 6(3):507-37.

Lyberg, Ingrid. 1984. Att Fråga om Barn: Teknisk Beskrivning av Intervjuundersökningen Kvinnor i Sverige, om Familj och Arbetsliv (To Ask about Children: Technical Description of the Interview Investigation Women in Sweden, Family and Career). Stockholm: Statistics Sweden.

Statistics Sweden. 1982. Population Changes 1981, Part 3: the Whole Country and the Counties etc. Stockholm: Statistics Sweden.

-. 1983. Population Projection for Sweden 1983-2025. Stockholm: Statistics Sweden.

-. 1989. The Future Population of Sweden: Projection for the Years 1989-2025. Stockholm: Statistics Sweden.

-. 1990. Statistical Abstract of Sweden 1991. Stockholm: Statistics Sweden.

UNS (United Nations Secretariat). 1982. Long-range global population projections, as assessed in 1980. Population Bulletin of the United Nations 14:17-30.

Williams, Linda B. and Basil G. Zimmer. 1990. The changing influence of religion on U.S. fertility: evidence from Rhode Island. Demography 27(3):475-481. 